\title{
CMOS Magnetic Field to Frequency Converter
}

\author{
Shr-Lung Chen, Chien-Hung Kuo, and Shen-Iuan Liu, Member, IEEE
}

\begin{abstract}
In this paper, a CMOS magnetic field to frequency converter with high resolution is presented. It is composed of two voltage-controlled ring oscillators whose output frequency differences linearly vary with the magnetic field perpendicular to the chip surface. The prototype circuit has been fabricated in a $0.5-\mu \mathrm{m}$ CMOS process and operated at a $5-\mathrm{V}$ supply voltage. The measured sensitivity is $24 \mathrm{kHz} / \mathrm{mT}$ and the power consumption is $5.1 \mathrm{~mW}$. The small equivalent resolution of at least $20 \mu \mathrm{T}$ can be achieved. The frequency offset is $42 \mathrm{kHz}$ when no magnetic field applied. Its nonlinearity within $\pm 120 \mathrm{mT}$ is smaller than $0.56 \%$.
\end{abstract}

Index Terms-C $\mathrm{C}^{2} \mathrm{MOS}$, Magnetic MOSFET (MAGFET), magnetooperational amplifier (MOP), magnetically controlled oscillator (MCO).

\section{INTRODUCTION}

A MAGNETIC sensor is a transducer which can convert a magnetic field into a corresponding electrical signal. There are a lot of industrial applications where magnetic sensors can be found [1], [2], such as brushless motor controls, current measurement, monitoring the exposure to magnetic field [3], automotive applications, etc. Integrated microsensors with on-chip interface circuits are currently replacing discrete sensors in view of their inherent advantages, such as low cost, high reliability, and on-chip processing capability. The output signal of a sensor can be converted into different forms depending on the circuit approaches used and system applications. To achieve small, robust, and low-cost micro systems, it is desirable to integrate the magnetic sensor with analog signal conditioning circuits so that their output can be fully compatible with digital signal processors. In this paper, a highly sensitive magnetic field sensor with frequency output is presented by using the magnetic MOSFET (MAGFET) [4] in a standard CMOS process. In this work, we integrate the magnetooperational amplifier (MOP) [5], [6] with two voltage-controlled ring oscillators to realize the magnetic field sensor.

This paper is organized as follows. In Section II, the magnetic field detection by the MOP is reviewed. Then, the magnetically-controlled delay element and the magnetically voltagecontrolled ring oscillator will be described. In Section III, the experimental results are given, and the conclusion is given in Section IV.

Manuscript received March 25, 2002; revised October 31, 2002. The associate editor coordinating the review of this paper and approving it for publication was Dr. Andre Bossche.

The authors are with the Department of Electrical Engineering, Graduate Institute of Electronics Engineering, National Taiwan University, Taipei, Taiwan 10617, R.O.C. (e-mail: 1si@cc.ee.ntu.edu.tw).

Digital Object Identifier 10.1109/JSEN.2002.807485

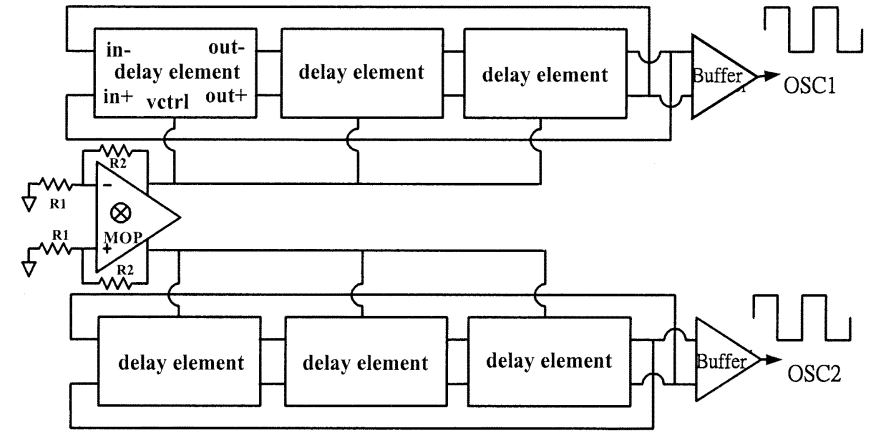

Fig. 1. Proposed magnetic sensor with frequency output.

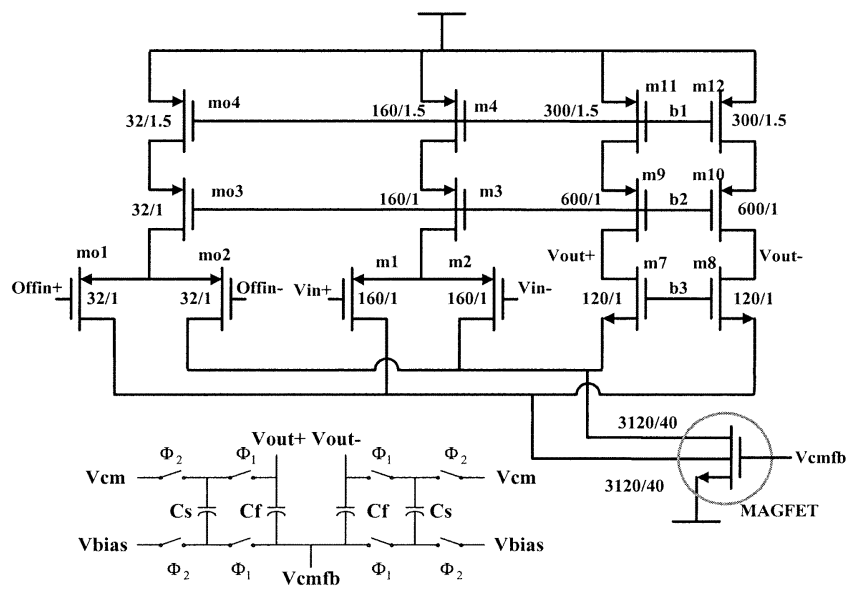

Fig. 2. Proposed CMOS magneto-operational amplifier.

\section{CiRCUIT DesCRIPTION}

The proposed magnetic field sensor is shown in Fig. 1. It is composed of the CMOS MOP [5], [6] and two voltage-controlled ring oscillators, which are controlled by the MOP. The schematic of the MOP with the switched-capacitor common mode feedback circuit is shown in Fig. 2. The MOP is a conventional folded-cascode amplifier except that the NMOS current sources are replaced by MAGFET arrays. Taking the layout mismatch problem and optimal sensitivity into account simultaneously, we choose the $n$ channel MAGFET as the sensing element and the aspect ratio of each MAGFET device in the following sections is chosen to be $W / L=80 \mu \mathrm{m} / 40 \mu \mathrm{m}$ and $d=2$, according to [6]. Since the bias current of the MAGFET is $700 \mu \mathrm{A}$, the power consumption of this chip mainly comes from the MOP. When no magnetic field is applied, the output drain currents of MAGFET are equal, which means that the voltage difference between the two differential outputs of the MOP is zero. In this situation, the MOP works just like a general operational amplifier. The additional differential pair, mol and mo2 in Fig. 2, is used to null the offset voltage. When the perpendicular 


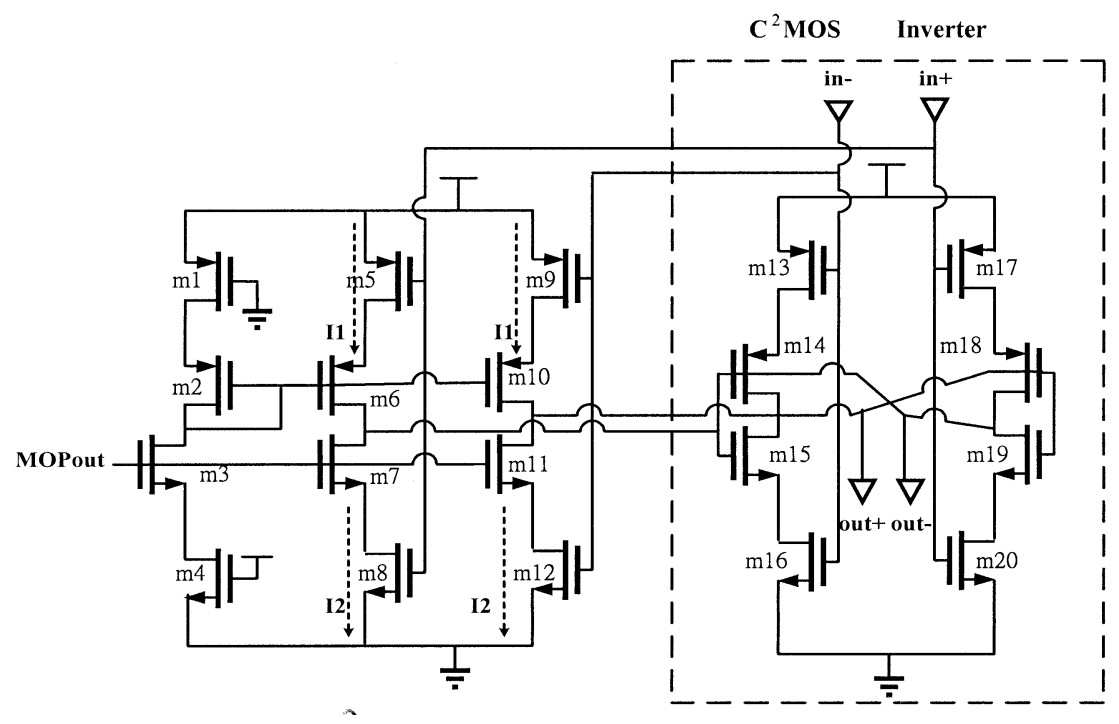

Fig. 3. Delay element.

magnetic field is applied, there is a current imbalance between these two drains. The voltage difference between the differential outputs $V_{\text {out }}$ and $V_{\text {out- }}$ will be generated. One can assume that a magnetic field induced voltage is added to the input of the MOP. So, the output voltage of the MOP can be expressed as

$$
V_{\text {out }+}-V_{\text {out- }}=A \cdot\left(V_{\text {in }+}-V_{\text {in- }}+v_{m}\right)
$$

where $A$ is the finite dc gain of this operational amplifier and $v_{m}\left(=S_{m} \cdot B_{\perp}\right)$ is the induced voltage. The conversion gain $S_{m}$ denotes the ratio between the induced voltage $v_{m}$ and the perpendicular magnetic field $B_{\perp}$. By connecting the MOP with resistors into an inverting amplifier, the linearity could be improved and the gain and sensitivity could be adjusted by the resistive ratio.

The voltage-controlled ring oscillator is composed of the magnetically controlled delay elements. This delay element is shown in Fig. 3 and it consists of two clocked CMOS $\left(C^{2} M O S\right)$ inverters [7], [8] with four switches ( $\mathrm{m} 6, \mathrm{~m} 7, \mathrm{~m} 10$, and $\mathrm{m} 11)$ and four current sources $(\mathrm{m} 5, \mathrm{~m} 8, \mathrm{~m} 9$, and $\mathrm{m} 12)$, which are generated by the outputs of the MOP. Two $C^{2} M O S$ inverters are interconnected to speed up the transition for the output swing between $V_{D D}-V_{T P}$ and $V_{T N}$, where $V_{D D}$ is the supply voltage and $V_{T P}$ and $V_{T N}$ are the threshold voltages of PMOS and NMOS transistors, respectively. Transistors, $\mathrm{m} 1$ and $\mathrm{m} 4$, are added to match the current sources with switches. The current source $I_{2}$ can be expressed as

$$
I_{2}=K\left(V_{G S 0}+r \cdot S_{m} \cdot B_{\perp}-V_{T N}\right)^{2}
$$

where $K$ is the transcoductance parameter, $V_{G S 0}$ is the original overdrive of the transistor driven by the MOP when no external magnetic field is applied, and $r\left(\equiv 1+\left(R_{2} / R_{1}\right)\right)$ is the voltage gain as shown in Fig. 1. The operation of the delay element can be described as follows [8].

If the In+ increases to $V_{D D}$ and in- decreases to ground, $\mathrm{m} 8$ is turned on ( $\mathrm{m} 5$ is turned off) and the current $I_{2}$ from $\mathrm{m} 7$ discharges out-; meanwhile, $\mathrm{m} 9$ is turned on ( $\mathrm{m} 12$ is turned off) and the current $I_{1}$ from m10 charges out + . In the beginning, out - is pulled down slowly by $I_{2}$, and $\mathrm{m} 14$ would not been turned on until out - is lower than $V_{D D}-V_{T P}$. Then, out+ will be charged to $V_{D D}$ quickly due the positive feedback of the $C^{2} M O S$ latch. In the same way, out+ increases slowly at first, and $\mathrm{m} 20$ would not be turned on until out+ increased higher than $V_{T N}$. Then, out+ will be discharged to ground quickly. The controlled current $I_{2}$ is used to discharge the output node from $V_{D D}$ to $V_{D D}-V_{T P}$. If the state transition time for this $C^{2} M O S$ latch can be neglected, the delay time $T_{D}$ of this delay element can be expressed as

$$
T_{D} \approx \frac{C \times V_{T P}}{I_{2}}
$$

where $C$ is the capacitive load of the output node. Two current sources $I_{1}$ are used to compensate the clock feed-through coupling because this clock feed-through would result in the distortion of the duty cycle [8]. In summary, this delay time of the delay element should mainly be contributed from $T_{D}$ and the time spending on the state transition in the regenerative latch could be neglected compared to $T_{D}$. The delay cell can provide wide delay range and negligible dc power dissipation.

The number of stages in a ring oscillator can be determined by various requirements, including speed, power dissipation, noise immunity, etc. By cascading odd $N$ delay elements in a loop, a ring oscillator can be formed; and its oscillating frequency can be given as

$$
f_{O S C}=\frac{1}{N \times T_{D}}
$$

where $T_{D}$ is the delay time of each delay element. We choose $N=3$ for compromise among power, speed and chip area.

However, the output frequency shown in (4) is a square term rather than a linear term related to $B_{\perp}$. To compensate the nonlinearity appear in (4), two ring oscillators are connected with the MOP in a fully differential manner as shown in Fig. 1. One can obtain the difference of the output frequencies by frequency counters to obtain a linear term. This structure has two advantages. One is that the sensitivity is doubled. The other is that the 


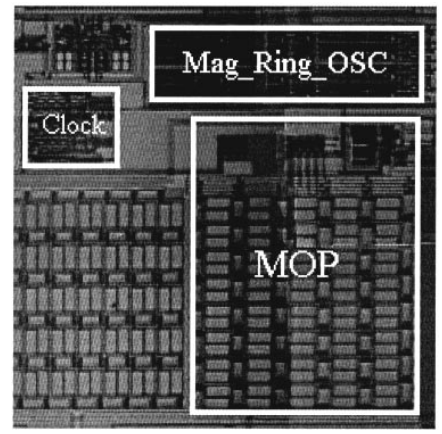

Fig. 4. Die photo of the proposed magnetic sensor.

even-order nonlinear terms can be cancelled if these two ring oscillators are well matched. The output frequency difference can be expressed as

$\Delta f=f_{O S C 1}-f_{O S C 2}=\frac{4 K \cdot r \cdot S_{m} \cdot B_{\perp}}{N \cdot C \cdot V_{T P}} \cdot\left(V_{G S 0}-V_{T N}\right)$.

From (5), the frequency difference relative to $B$ can be maximized by adjusting various variables such as $N, C, r$, and $V_{G S O}$. The fully differential structure of the MOP and the ring oscillators can not only increase the sensitivity, but also minimize some sensor defects like offset and nonlinearity. Moreover, the MOP increases the sensitivity of the MAGFET device by the factor of the feedback resistor ratio, and the MOP is integrated with the ring oscillators to produce a frequency-modulated signal which is highly immune to noise and interferences.

\section{EXPERIMENTAL RESULTS AND DISCUSSIONS}

The sensor has been fabricated in a $0.5-\mu \mathrm{m}$ CMOS technology with a 5-V supply voltage. The die photo of the proposed magnetic field to frequency converter is shown in Fig. 4, and the total area is $1.38 \mathrm{~mm}^{*} 1 \mathrm{~mm}$ excluding the $\mathrm{I} / \mathrm{O}$ pads. The off-chip feedback resistors for the MOP is $R_{1}=1 \mathrm{k} \Omega$ and $R_{2}=220 \mathrm{k} \Omega$, respectively. The buffers of the oscillators are two inverters made on chip for driving the external load. The magnetic field perpendicular to the chip is generated by an electromagnet core which is powered by the GW regulated dc power supply (Model GPR-22H10H). The current supplied by this power supply controls the magnitude and polarity of the generated field. The generated field is checked by the Gauss/Tesla meter (F.W. BELL model 5080) with a Hall probe closely approximating to the test chip. The above mentioned equipment and devices are caged in an iron shell. Although this cannot shield the Earth's magnetic field from introducing offset frequency, it can prevent other electric effects from the air as completely as possible. The remedy for the offset induced by the Earth itself is to shield the Hall probe in the zero flux chamber of Gauss/Tesla meter before our measurement stage. The output frequency of the magnetically controlled oscillator (MCO) was studied as a function of the dc magnetic induction, and these two output signals from two oscillators are connected to the frequency counter to fulfill the subtraction.

The measured output frequency differences are shown in Figs. 5 and 6 . Each measured data is obtained by averaging five measurements. The coarse measurement result is plotted in

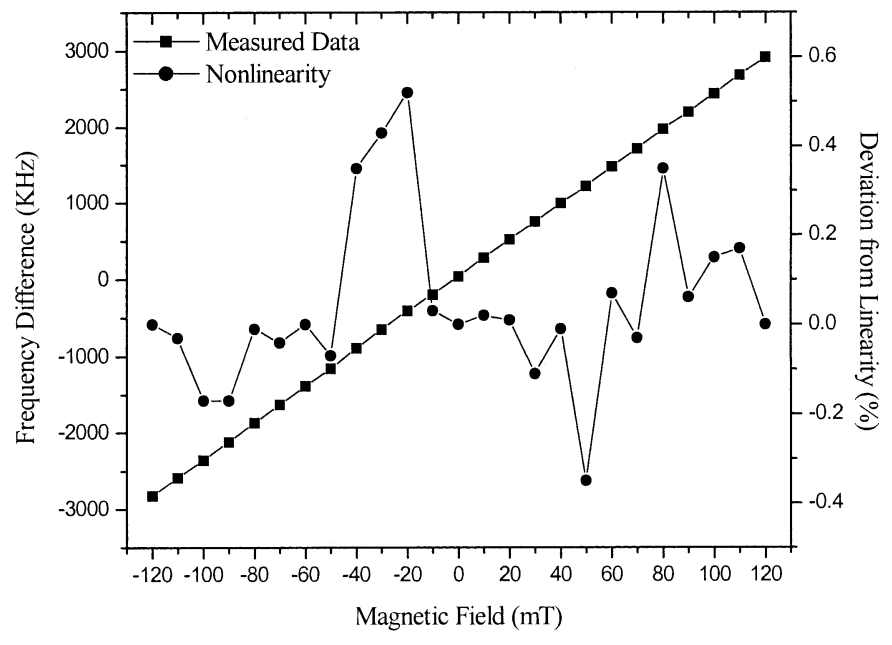

Fig. 5. Coarse measurement result.

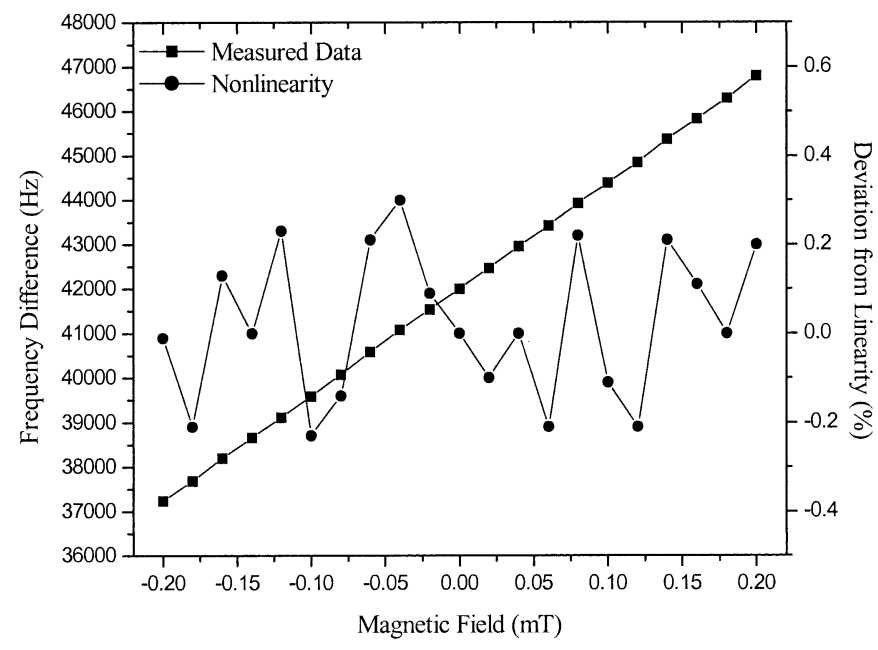

Fig. 6. Fine measurement result.

TABLE I

PERFORMANCE SUMMARY OF THE PROPOSED MAGNETIC FIELD TO FREQUENCY CONVERTER

\begin{tabular}{l|l}
\hline Range & $\pm 120 \mathrm{mT}$ \\
\hline Offset frequency & $42 \mathrm{kHz}$ \\
\hline Resolution & $<20 \mathrm{uT}$ \\
\hline Sensitivity (kHz/mT) & $\begin{array}{l}27 \text { (simulation) } \\
24 \text { (measurement) }\end{array}$ \\
\hline
\end{tabular}

Fig. 5, where the range is within $\pm 120 \mathrm{mT}$ and the magnitude incremental is $10 \mathrm{mT}$. From this plot, the linear response owing to the magnetic field can be obtained. The calculated sensitivity is $24 \mathrm{kHz} / \mathrm{mT}$. Fig. 6 shows the resolution test. The linearity is also maintained in this range of $\pm 0.2 \mathrm{mT}$ with $20 \mu \mathrm{T}$ increment. From this plot, we can deduce that the real resolution should be much smaller. Restricted by the accuracy of the instruments, the change of the magnetic filed smaller than $20 \mu \mathrm{T}$ could not be generated for further verifications. Since only the dc magnetic field signal is tested, the cut off frequency for the magnetic 
TABLE II

Performance COMPARISONS With PUBLISHEd WORKS

\begin{tabular}{l|l|l|l|l|l}
\hline & Proposed & {$[10]$} & {$[11]$} & {$[12]$} & {$[13]$} \\
\hline Range $(\mathrm{mT})$ & $\pm 120 \mathrm{mT}$ & $\pm 400 \mathrm{mT}$ & $\pm 1000 \mathrm{mT}$ & $0 \sim 10 \mathrm{mT}$ & $0.2-2.6 \mathrm{mT}$ \\
\hline Sensitivity $(\mathrm{Hz} / \mathrm{mT})$ & 24015 & 200 & 0.184 & 3 & 79 \\
\hline Linearity & $0.56 \%$ & $0.1 \%$ & $0.1 \%$ & $10 \%$ & $5.6 \%$ \\
\hline Resolution & $<20 \mathrm{uT}$ & $<5 \mathrm{mT}$ & Not mentioned & $100 \mathrm{uT}$ & $400 \mathrm{uT}$ \\
\hline VDD & $5 \mathrm{~V}$ & $1 \mathrm{~V}$ & $10 \mathrm{~V}$ & $2 \mathrm{~V}$ & $5 \mathrm{~V}$ \\
\hline $\begin{array}{l}\text { Power } \\
\begin{array}{l}\text { Figure of Merit }= \\
\text { sensitivity }(H z / m T)\end{array}\end{array}$ & $5.1 \mathrm{~mW}$ & $1.2 \mathrm{~mW}$ & $6.2 \mathrm{~mW}$ & $0.2 \mathrm{uW}$ & $135 \mathrm{uW}$ \\
\hline $\begin{array}{l}\text { power }(m W) \bullet \text { linearity }(\%) \\
\text { Process }\end{array}$ & 8408.6 & 1666.7 & 0.3 & 1500 & 104.5 \\
\hline
\end{tabular}

signal should be low. The minimum detectable magnetic field of the proposed circuit under dc excitation is limited by $1 / f$ noise and not by thermal noise.

In Fig. 6, the output frequency with no magnetic field corresponds to the center frequency of $42 \mathrm{kHz}$. This offset phenomenon can be caused from mismatch from the MOP and any mismatch between these two oscillators. The performance summary is given in Table I. This sensor's nonlinearity is less than $0.56 \%$ for the magnetic field within $\pm 120 \mathrm{mT}$. The power consumption of the proposed magnetic sensor is $5.1 \mathrm{~mW}$. The measured sensitivity $(24 \mathrm{kHz} / \mathrm{mT})$ shows the degradation compared with the simulation result $(27 \mathrm{kHz} / \mathrm{mT})$. The nonlinearity problem may be caused by any mismatch between the differential paths and temperature effects. For example, there is a mismatch between the resistors $\mathrm{R} 1$ and $\mathrm{R} 2$ in the MOP or mismatch in the output capacitances of the delay elements, and the even order nonlinear terms can not be completely cancelled like in (5).

The temperature characteristics of the chip are verified from HSPICE simulations. The temperature impact on output frequency variations is examined under different process corner variations and different frequencies. The largest frequency variations are $8.32 \%$ under four different process corners and $8.57 \%$ during the range of $20-40{ }^{\circ} \mathrm{C}$. These values correspond approximately to $5000 \mathrm{ppm} /{ }^{\circ} \mathrm{C}$ and agree exactly with that of MAGFET reported ain [9].

Performance comparisons with published works using MAGFETs are listed in Table II. Our work can give the improvement in sensitivity over previously reported works using MAGFETs. Moreover, a figure of merit is defined as $(\operatorname{sensitivity}(\mathrm{Hz} / \mathrm{mT})) /(\operatorname{power}(\mathrm{mW})$ - linearity $(\%))$ and our work can achieve the best figure of merit in Table II.

\section{CONCLUSION}

A highly sensitive magnetic field sensor with the sensitivity of $24 \mathrm{KHz} / \mathrm{mT}$ is presented. This sensor can detect the magnetic fields smaller than $20 \mu \mathrm{T}$. Compared with the published works with frequency output by using MAGFETs, it can exhibit a good figure of merit and the fine resolution according to Table II. The frequency output can be further processed by digital signal processing circuits, such as a microcontroller, to achieve the high resolutions.

\section{ACKNOWLEDGMENT}

The authors would like to thank the Chip Implementation Center (CIC) of the National Science Council, Taiwan, R.O.C., for fabricating the chips.

\section{REFERENCES}

[1] C. Schott, F. Burger, H. Blanchard, and K. Chiesi, "Modern integrated silicon hall sensors," Sens. Rev., vol. 18, pp. 252-257, 1998.

[2] P. Ripka, "Magnetic sensors for industrial and field applications," Sens. Actuators A, vol. 41-42, pp. 394-397, 1994.

[3] P. Malcovati and F. Maloberti, "An integrated microsystem for 3-D magnetic field measurements," IEEE Trans. Instrum. Measurement, pp. 341-345, Apr. 2000.

[4] H. Baltes and R. Popovic, "Integrated semiconductor magnetic field sensors," Proc. IEEE, vol. 74, pp. 1107-1132, Aug. 1986.

[5] K. Maenaka, H. Okada, and T. Nakamura, "Universal magneto-operational amplifier (MOP)," Sens. Actuators A, vol. 21-23, pp. 807-811, 1990.

[6] S. I. Liu, J. F. Wei, and G. M. Sung, "SPICE macro model for MAGFET and its applications," IEEE Trans. Circuits Syst. II, vol. 46, pp. 370-375, Apr. 1999.

[7] N. Weste and K. Eshraghian, Principles of VLSI Design: A Systems Perspective, 2nd ed. Reading, MA: Addison-Wesley, 1993.

[8] K. H. Chen, L. J. Tzou, and H. S. Liao, "A low-jitter and low-power phase-locked loop design," in Proc. IEEE ISCAS, May 2000, pp. 257-260.

[9] J. Ryan, J. Doyle, M. Buckley, and M. Flynn, "A magnetic field sensitive amplifier with temperature compensation," in Proc. IEEE Int. SolidState Circuits Conf., Feb. 1992, pp. 124-125.

[10] S. Gumenjuk and B. Podlepetsky, "Advanced integrated magnetic-field sensors with frequency output," Sens. Actuators A, vol. 62, pp. 529-533, 1997.

[11] A. Natyhan, I. A. Mckay, and I. M. Filanovsky, "Design of a CMOS oscillator with magnetic-field frequency modulation," IEEE J. Solid-State Circuits, vol. SC-22, Apr. 1987.

[12] J. Doyle and C. Lyden, "High sensitivity low power silicon magnetic field detector," in Proc. IEEE Custom Integrated Circuits Conf., May 1994, pp. 274-277.

[13] J. Doyle, "High sensitivity silicon magnetic field detection," in Proc. IEEE Custom Integrated Circuits Conf., May 2001, pp. 105-108. 


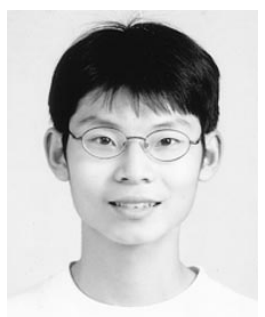

Shr-Lung Chen was born in Taipei, Taiwan, R.O.C., on November 8, 1976. He received the B.S. and M.S. degrees in electrical engineering from National Taiwan University, Taipei, in 1999 and 2001, respectively. He is currently applying to graduate schools in the U.S. to pursue the Ph.D. degree.

He is currently a Second Lieutenant (Special Electrical Engineering Officer) with the Communication Development Office, Ministry of National Defense, R.O.C. His research interests include analog integrated circuit designs, especially magnetic sensors actuators and delta-sigma modulators.

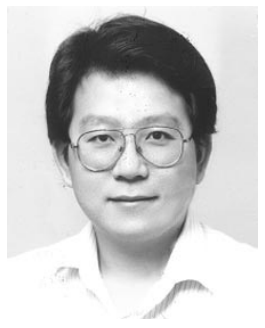

Chien-Hung Kuo was born in Taipei, Taiwan, R.O.C., on April 1, 1965. He received the B.S. degree in electrical engineering from Feng Chia University, Taiwan, in 1987, and the M.S. degree in electrical engineering from the National Taiwan University (NTU), Taipei, in 1992. He is currently pursuing the Ph.D. degree in the Department of Electrical Engineering, NTU.

His research focuses on the design of analog-to-digital conversions, including pipelined lators

analog-to-digital converters and delta-sigma modu-

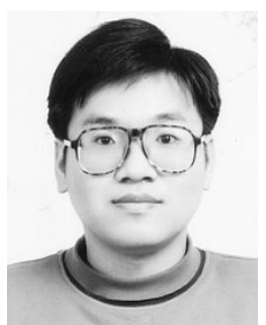

Shen-Iuan Liu (S'88-M'93) was born in Keelung, Taiwan, R.O.C., on April 4, 1965. He received the B.S. and $\mathrm{Ph} . \mathrm{D}$. degrees in electrical engineering from the National Taiwan University (NTU), Taipei, in 1987 and 1991, respectively.

During 1991-1993, he served as a Second Lieutenant in the Chinese Air Force. During 1991-1994, he was an Associate Professor in the Department of Electronic Engineering, National Taiwan Institute of Technology. He joined the Department of Electrical Engineering, NTU, in 1994, and he has been a Professor since 1998. His research interests are in analog and digital integrated circuits and systems. 